\title{
Analysis of Control Systems on Symmetric Cones
}

\author{
Ivan Papusha Richard M. Murray
}

\begin{abstract}
It is well known that exploiting special structure is a powerful way to extend the reach of current optimization tools to higher dimensions. While many linear control systems can be treated satisfactorily with linear matrix inequalities (LMI) and semidefinite programming (SDP), practical considerations can still restrict scalability of general methods. Thus, we wish to work with high dimensional systems without explicitly forming SDPs. To that end, we exploit a particular kind of structure in the dynamics matrix, paving the way for a more efficient treatment of a certain class of linear systems. We show how second order cone programming (SOCP) can be used instead of SDP to find Lyapunov functions that certify stability. This framework reduces to a famous linear program (LP) when the system is internally positive, and to a semidefinite program (SDP) when the system has no special structure.
\end{abstract}

\section{INTRODUCTION}

This paper is concerned with stability of the linear dynamical system

$$
\dot{x}(t)=A x(t), \quad A \in \mathbf{R}^{n \times n}, \quad x(t) \in \mathbf{R}^{n},
$$

under certain conditions on the dynamics matrix $A$. When this matrix has no special structure, the system is stable if and only if there exists a symmetric matrix $P=P^{T} \in \mathbf{R}^{n \times n}$ satisfying the linear matrix inequalities

$$
P=P^{T} \succ 0, \quad A^{T} P+P A \prec 0 .
$$

The existence of such a matrix $P$ corresponds to the existence of a quadratic Lyapunov function $V: \mathbf{R}^{n} \rightarrow \mathbf{R}$,

$$
V(x)=\langle x, P x\rangle,
$$

which is positive definite $(V(x)>0$ for all $x \neq 0)$ and decreasing $(\dot{V}<0$ along trajectories of (1)).

To find a matrix $P$ that satisfies (2), one generally needs to formulate and solve a semidefinite program (SDP), however, if $A$ has special structure, this semidefinite program can sometimes be cast as a simpler linear program (LP)—with advantages in numerical stability, opportunities for parallelism, and better scaling to high dimensions $(n \gg 1)$.

For example, if $A$ is a given Metzler matrix, i.e., its offdiagonal entries are nonnegative,

$$
A_{i j} \geq 0, \quad \text { for all } i \neq j,
$$

then it suffices to search for a diagonal matrix $P$ satisfying (2). Equivalently, condition (2) can be replaced with the simpler vector condition

$$
p_{i}>0, \quad(A p)_{i}<0, \quad \text { for all } i=1, \ldots, n \text {. }
$$

The authors are with the Control and Dynamical Systems Department, California Institute of Technology, Pasadena, CA USA.
Finding a vector $p \in \mathbf{R}^{n}$ that satisfies this second condition (3) can be formulated as an LP with fewer decision variables than the corresponding SDP [1], [2], [3].

Systems for which $A$ is a Metzler matrix are called internally positive, because their state stays in the nonnegative orthant $\mathbf{R}_{+}^{n}$ if it starts in the nonnegative orthant. As we will see, the Metzler structure is (in a certain sense) the only natural matrix structure for which an LP may generically be composed to verify stability. However, the inclusion

$$
\mathrm{LP} \subseteq \mathrm{SOCP} \subseteq \mathrm{SDP}
$$

interpreted as an expressiveness ranking of popular conic programming methods, begs the question of whether stability analysis can be cast, for example, as a second order cone program (SOCP) for some specific subclass of linear dynamics, in the same way that it can be cast as an LP for internally positive systems, and an SDP for unstructured linear systems.

More generally, we wish to know when certain conic programming techniques can be used to verify stability of certain linear systems. In answering this question we will discuss a generalization of the Metzler property known as cross-positivity and the notion of a Jordan algebra to characterize dynamics that are exponentially invariant with respect to a symmetric cone. In so doing, we demonstrate the strong unifying power of the Jordan product on linear systems and discuss a rich, little-known class of systems that admit SOCP-based analysis.

\section{JORDAN AlgEBRAS AND SYMMETRIC CONES}

The following background is informal and meant to set out notation, adopting conventions familiar to system theory and optimization [4], [5], [6]. Jordan algebraic techniques have proved to be effective in unifying interior point methods for conic programming [7]. In the context of symmetric cones, they are are rich and well studied field, with an excellent reference [8]. For more recent reviews, see [9], [10].

\section{A. Cones on a vector space.}

Let $V$ be a vector space over the reals with inner product $\langle\cdot, \cdot\rangle$. A subset $K \subseteq V$ is called a cone if it is closed under nonnegative scalar multiplication: for every $x \in K$ and $\theta \geq 0$ we have $\theta x \in K$. A cone $K$ is pointed if it contains no line, or equivalently

$$
x \in K,-x \in K \quad \Longrightarrow \quad x=0 .
$$

A cone is proper if it is closed, convex, pointed, and has nonempty interior. Every proper cone induces a partial order $\preceq$ on $V$ given by

$$
x, y \in V, \quad x \preceq y \quad \Longleftrightarrow \quad y-x \in K .
$$


We have $x \prec y$ if and only if $y-x \in \operatorname{int} K$. Similarly we write $x \succeq y$ and $x \succ y$ to mean $y \preceq x$ and $y \prec x$, respectively.

\section{B. Jordan algebras.}

A (Euclidean) Jordan algebra is an inner product space $(V,\langle\cdot, \cdot\rangle)$ endowed with a Jordan product $\circ: V \times V \rightarrow V$, which satisfies the following properties:

1) Bilinearity: $x \circ y$ is linear in $x$ for fixed $y$ and vice-versa

2) Commutativity: $x \circ y=y \circ x$

3) Jordan identity: $x^{2} \circ(y \circ x)=\left(x^{2} \circ y\right) \circ x$

4) Adjoint identity: $\langle x, y \circ z\rangle=\langle y \circ x, z\rangle$.

Note that a Jordan product is commutative, but it need not be associative. When we interpret $x^{2}=x \circ x$, the Jordan identity allows any power $x^{k}, k \geq 2$ to be inductively defined. An identity element e satisfies $\mathbf{e} \circ x=x \circ \mathbf{e}=x$ for all $x \in V$, and defines a number $r=\langle\mathbf{e}, \mathbf{e}\rangle$ called the rank of $V$.

The cone of squares $K$ corresponding to the Jordan product $\circ$ is defined as

$$
K=\{x \circ x \mid x \in V\} .
$$

Every element $x \in V$ has a spectral decomposition

$$
x=\sum_{i=1}^{r} \lambda_{i} e_{i}
$$

where $\lambda_{i} \in \mathbf{R}$ are eigenvalues of $x$ and the set of eigenvectors $\left\{e_{1}, \ldots, e_{r}\right\} \subseteq V$, called a Jordan frame, satisfies

$$
e_{i}^{2}=e_{i}, \quad e_{i} \circ e_{j}=0 \text { for } i \neq j, \quad \sum_{i=1}^{r} e_{i}=\mathbf{e} .
$$

We have $x \in K$ (written $x \succeq_{K} 0$, or simply $x \succeq 0$ when the context is clear) provided $\lambda_{i} \geq 0$. Similarly, $x \in$ int $K$ if and only if $\lambda_{i}>0$ (written $x \succ_{K} \quad 0$ ). The spectral decomposition allows us to define familiar concepts like trace, determinant, and square root of an element $x$ by taking the corresponding function of the eigenvalue. That is,

$$
\operatorname{tr} x=\sum_{i=1}^{r} \lambda_{i}, \quad \operatorname{det} x=\prod_{i=1}^{r} \lambda_{i}, \quad x^{1 / 2}=\sum_{i=1}^{r} \lambda_{i}^{1 / 2} e_{i},
$$

where the last quantity only makes sense if $x \succeq 0$.

Finally, every element $z \in V$ has a quadratic representation, which is a map $P_{z}: V \rightarrow V$ given by

$$
P_{z}(x)=2(z \circ(z \circ x))-z^{2} \circ x .
$$

\section{Symmetric cones.}

The closed dual cone of $K$ is defined as

$$
K^{*}=\{y \in V \mid\langle x, y\rangle \geq 0, \text { for all } x \in K\},
$$

A cone $K$ is self-dual if $K^{*}=K$. The automorphism group $\operatorname{Aut}(K)$ of an open convex cone $K$ is the set of invertible linear transformations $g: V \rightarrow V$ that map $K$ to itself,

$$
\operatorname{Aut}(K)=\{g \in G L(V) \mid g K=K\} .
$$

An open cone $K$ is homogeneous if $\operatorname{Aut}(K)$ acts on the cone transitively, in other words, if for every $x, y \in K$ there exists $g \in \operatorname{Aut}(K)$ such that $g x=y$. Finally, $K$ is symmetric if it is homogeneous and self-dual. Symmetric cones are an important object of study because they are the cones of squares of a Jordan product, admit a spectral decomposition, and are (in finite dimensions, [8]) isomorphic to a Cartesian product of

- $n \times n$ self-adjoint positive semidefinite matrices with real, complex, or quaternion entries,

- $3 \times 3$ self-adjoint positive semidefinite matrices with octonion entries (Albert algebra), and

- Lorentz cone.

In this work, we pay attention to symmetric cones because they have a differentiable log-det barrier function, allowing numerical optimization with interior point methods [7], [11]. The symmetric cones $\mathbf{R}_{+}^{n}\left(=\mathbf{S}_{+}^{1} \times \cdots \times \mathbf{S}_{+}^{1}\right), \mathcal{L}_{+}^{n}$, and $\mathbf{S}_{+}^{n}$ give rise to LP, SOCP, and SDP, respectively. We now define these three cones.

\section{Examples of symmetric cones.}

1) Nonnegative orthant $\mathbf{R}_{+}^{n}$ : the cone of squares associated with the standard Euclidean space $\mathbf{R}^{n}$ and Jordan product

$$
(x \circ y)_{i}=x_{i} y_{i}, \quad i=1, \ldots, n,
$$

i.e., the entrywise (or Hadamard) product. The identity element is $\mathbf{e}=\mathbf{1}=(1, \ldots, 1)$, and the Jordan frame comprises the standard basis vectors. The quadratic representation of an element $z \in \mathbf{R}^{n}$ is given by the diagonal matrix $P_{z}=$ $\operatorname{diag}(z)^{2}$

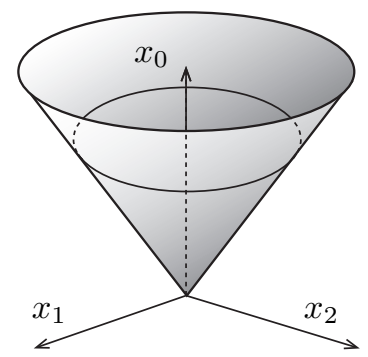

Fig. 1. Second-order (Lorentz) cone $\mathcal{L}_{+}^{3}$.

2) Lorentz cone $\mathcal{L}_{+}^{n}$ : partition every element of $\mathbf{R}^{n}$ as $x=\left(x_{0}, x_{1}\right) \in \mathbf{R} \times \mathbf{R}^{n-1}$ and define the Lorentz cone (also known as second-order or norm cone) as

$$
\mathcal{L}_{+}^{n}=\left\{\left(x_{0}, x_{1}\right) \in \mathbf{R} \times \mathbf{R}^{n-1} \mid\left\|x_{1}\right\|_{2} \leq x_{0}\right\} \subseteq \mathbf{R}^{n} .
$$

This cone is illustrated in Figure 1 for the case $n=3$. It can be shown that $\mathcal{L}_{+}^{n}$ is the cone of squares corresponding to the Jordan product

$$
\left[\begin{array}{l}
x_{0} \\
x_{1}
\end{array}\right] \circ\left[\begin{array}{l}
y_{0} \\
y_{1}
\end{array}\right]=\left[\begin{array}{c}
\langle x, y\rangle \\
x_{0} y_{1}+y_{0} x_{1}
\end{array}\right]
$$

The rank of this algebra is 2 , giving a particularly simple spectral decomposition for a given element $x$,

$$
x=\lambda_{1} e_{1}+\lambda_{2} e_{2},
$$


where the eigenvalues and Jordan frame are

$$
\begin{aligned}
\lambda_{1} & =x_{0}+\left\|x_{1}\right\|_{2}, & \lambda_{2} & =x_{0}-\left\|x_{1}\right\|_{2}, \\
e_{1} & =\frac{1}{2}\left[\begin{array}{c}
1 \\
x_{1} /\left\|x_{1}\right\|_{2}
\end{array}\right], & e_{2} & =\frac{1}{2}\left[\begin{array}{c}
1 \\
-x_{1} /\left\|x_{1}\right\|_{2}
\end{array}\right] .
\end{aligned}
$$

The identity element is $\mathbf{e}=(1,0)$ and the quadratic representation of an element $z \in \mathbf{R}^{n}$ is the matrix

$$
P_{z}=z z^{T}-\frac{z^{T} J_{n} z}{2} J_{n},
$$

where $J_{n}=\operatorname{diag}(1,-1, \ldots,-1) \in \mathbf{R}^{n \times n}$ is the $n \times n$ inertia matrix with signature $(1, n-1)$. Note that $P_{z}$ is a positive semidefinite matrix if and only if $z \succeq \mathcal{L}_{+}^{n} 0$.

3) Positive semidefinite cone $\mathbf{S}_{+}^{n}$ : consider the vector space $\mathbf{S}^{n}$ of real, symmetric $n \times n$ matrices with the trace inner product. If we define the Jordan product as the symmetrized matrix product,

$$
X \circ Y=\frac{1}{2}(X Y+Y X)
$$

then the cone of squares is $\mathbf{S}_{+}^{n}$, the positive semidefinite matrices with real entries. The spectral decomposition of an element $X \in \mathbf{S}^{n}$ follows from the eigenvalue decomposition

$$
X=\sum_{i=1}^{n} \lambda_{i} v_{i} v_{i}^{T}, \quad \Longrightarrow \quad e_{i}=v_{i} v_{i}^{T}, \quad i=1, \ldots, n .
$$

The quadratic representation of an element $Z \in \mathbf{S}^{n}$ is given by the map $P_{Z}: \mathbf{S}^{n} \rightarrow \mathbf{S}^{n}$,

$$
P_{Z}(X)=2(Z \circ(Z \circ X))-(Z \circ Z) \circ X=Z X Z \text {. }
$$

\section{A LINK TO QUADRATIC LYAPUNOV FUNCTIONS}

Instead of discussing the original dynamics (1) on $\mathbf{R}^{n}$, we consider linear dynamics on a Jordan algebra $V$. This will allow us to present a unified treatment of the cones $\mathbf{R}_{+}^{n}, \mathcal{L}_{+}^{n}$, as well as restate familiar results from classical state space theory by specializing to $\mathbf{S}_{+}^{n}$.

\section{A. Cross-positive linear operators.}

A linear operator $L: V \rightarrow V$ is cross-positive with respect to a proper cone $K$ if

$$
x \in K, y \in K^{*} \text {, and }\langle x, y\rangle=0 \quad \Longrightarrow \quad\langle L(x), y\rangle \geq 0 .
$$

Exponentials of cross-positive operators leave the cone invariant: if $L: V \rightarrow V$ is cross-positive with respect to $K$, then $e^{t L}(K) \subseteq K$ for all $t \geq 0$. Interestingly, the converse is also true [12, Theorem 3].

For example, a matrix $A \in \mathbf{R}^{n \times n}$, thought of as a linear transformation $A: \mathbf{R}^{n} \rightarrow \mathbf{R}^{n}$, is cross-positive with respect to the cone $\mathbf{R}_{+}^{n}$ if and only if $A$ is a Metzler matrix, i.e.,

$$
A_{i j} \geq 0, \quad \text { for all } i \neq j,
$$

or equivalently trajectories $x(t)$ of the system

$$
\dot{x}(t)=A x(t)
$$

remain in $\mathbf{R}_{+}^{n}$ whenever they enter $\mathbf{R}_{+}^{n}$. Therefore, crosspositivity is a generalization of the Metzler property.

Examples of operators $L$ that satisfy $e^{L}(K) \subseteq K$ are
- Nonnegative orthant: $L(x)=A x$, where $A \in \mathbf{R}^{n \times n}$ is Metzler.

- Lorentz cone: $L(x)=A x$, where $A^{T} J_{n}+J_{n} A \succeq \xi J_{n}$ for some $\xi \in \mathbf{R}$.

- Positive semidefinite matrices: $L(X)=A X+X A^{T}$ for any matrix $A$.

In fact, the examples above precisely characterize all crosspositive operators on $\mathbf{R}_{+}^{n}$ and $\mathcal{L}_{+}^{n}$ (but not $\mathbf{S}_{+}^{n}$ ) [13].

\section{B. A class of Lyapunov functions}

Let $L: V \rightarrow V$ be a linear operator, and consider the linear dynamical system

$$
\dot{x}(t)=L(x), \quad x(0)=x_{0},
$$

where $x_{0} \in V$ is an initial condition. We make the assumption that $e^{L}(K) \subseteq K$, or equivalently, that $L$ is crosspositive. Systems that obey this this assumption are, of course, very special, because any trajectory that starts in the cone of squares $K$ will remain within $K$ for all time $t \geq 0$,

$$
x_{0} \in K \quad \Longrightarrow \quad x(t) \in K \text { for all } t \geq 0,
$$

in other words, such systems are exponentially invariant with respect to the cone $K$. We consider a generalization of the class of quadratic Lyapunov functions on $V$ given by

$$
V_{z}: V \rightarrow \mathbf{R}, \quad V_{z}(x)=\left\langle x, P_{z}(x)\right\rangle,
$$

where $z \in V$ is a parameter, and $P_{z}$ is the quadratic representation of $z$ in the Jordan algebra $V$. The following theorem gives a necessary and sufficient condition that $V_{z}$ is a Lyapunov function [9], [10], [14].

Theorem 1 (Gowda et al. 2009). Let $L: V \rightarrow V$ be a linear operator on a Jordan algebra $V$ with corresponding symmetric cone of squares $K$, and assume that $e^{L}(K) \subseteq K$. The following statements are equivalent:

(a) There exists $p \succ_{K} 0$ such that $-L(p) \succ_{K} 0$

(b) There exists $z \succ_{K} 0$ such that $L P_{z}+P_{z} L^{T}$ is negative definite on $V$.

(c) The system $\dot{x}(t)=L(x)$ with initial condition $x_{0} \in K$ is asymptotically stable.

Proof. See [14, Theorem 11].

This result allows the existence of a distinguished element $p \succ_{K} 0$ in the interior of $K$, where the vector field points in the direction of $-K$, or equivalently $-L(p) \succ_{K} 0$, to certify the existence of a quadratic Lyapunov function of the form

$$
V_{z}: V \rightarrow \mathbf{R}, \quad V_{z}(x)=\left\langle x, P_{z}(x)\right\rangle .
$$

In fact, the derivative of $V_{z}$ along trajectories of (4) is

$$
\begin{aligned}
\dot{V}_{z}(x) & =\left\langle\dot{x}, P_{z}(x)\right\rangle+\left\langle x, P_{z}(\dot{x})\right\rangle \\
& =\left\langle L(x), P_{z}(x)\right\rangle+\left\langle x, P_{z}(L(x))\right\rangle \\
& =\left\langle x, L^{T}\left(P_{z}(x)\right)\right\rangle+\left\langle x, P_{z}(L(x))\right\rangle \\
& =\left\langle x,\left(P_{z} L+L^{T} P_{z}\right)(x)\right\rangle
\end{aligned}
$$

For the algebra $\mathbf{R}^{n}$ and the corresponding cone $\mathbf{R}_{+}^{n}$, Theorem 1 translates as follows. The quadratic representation 
of an element $z \succ_{\mathbf{R}_{+}^{n}} 0$ is a diagonal matrix $D$ with strictly positive diagonal. Let $A$ be a cross-positive (i.e., Metzler) matrix. The system $\dot{x}(t)=A x(t)$ is stable (i.e., Hurwitz), if and only if there exists an entrywise positive vector $p$ such that

$$
p \succ_{\mathbf{R}_{+}^{n}} 0, \quad-A p \succ_{\mathbf{R}_{+}^{n}} 0
$$

which happens if and only if there exists a diagonal Lyapunov function $V(x)=x^{T} D x$,

$$
D \succ \mathbf{S}_{+}^{n} 0, \quad A D+D A^{T} \prec \mathbf{S}_{+}^{n} 0 .
$$

Finding such a $p$ is an LP for a fixed $A$. In addition, if we know (or impose, through a feedback interconnection) that $A$ has the Metzler structure, then a diagonal Lyapunov function candidate suffices to ensure stability. This trick has been used in, e.g., [3] to greatly simplify (and in certain cases parallelize) stability analysis and controller synthesis.

Now consider the algebra $\mathbf{S}^{n}$ and the corresponding cone $\mathbf{S}_{+}^{n}$. One can define many cross-positive operators, but one comes to mind: the Lyapunov transformation $L: \mathbf{S}^{n} \rightarrow \mathbf{S}^{n}$,

$$
L(X)=A X+X A^{T},
$$

where $A \in \mathbf{R}^{n}$ is a given matrix. (By construction, $L$ is cross-positive). Following the theorem, we now restate some widely known facts about linear systems. The matrix differential equation

$$
\dot{X}(t)=A X+X A^{T}, \quad X(0)=X_{0} \succ_{\mathbf{S}_{+}^{n}} 0
$$

is asymptotically stable if and only if there exists a matrix $P \succ 0$ such that $L(P)=A P+P A^{T} \prec 0$, if and only if there exists a matrix $Z \succ 0$ such that the function

$$
V_{Z}(X)=\left\langle X, P_{Z}(X)\right\rangle=\operatorname{Tr}(X Z X Z)=\|X Z\|_{F}^{2}
$$

is a Lyapunov function. This happens if and only if $A$ is a (Hurwitz) stable matrix.

\section{DYNAMICS ON A LORENTZ CONE}

The cones $\mathbf{R}_{+}^{n}$ and $\mathbf{S}_{+}^{n}$ have been well studied in the literature. The "intermediate case" - the cone $\mathcal{L}_{+}^{n}$-is, however, quite strange. This cone has received relatively little attention in the control community. Recent efforts have been in the context of model matching [15]. Our work here can be seen as a complement.

\section{A. Enforcing $\mathcal{L}_{+}^{n}$-invariance}

To apply the main theorem we require that $e^{L}(K) \subseteq K$. For the case $K=\mathcal{L}_{+}^{n}$, this means $A$ must satisfy the LMI

$$
A^{T} J_{n}+J_{n} A-\xi J_{n} \succeq 0, \quad \xi \in \mathbf{R} .
$$

If $A$ were to affinely depend on optimization variables (as it would if it were a closed loop matrix in a linear feedback synthesis problem), then enforcing $\mathcal{L}_{+}^{n}$-invariance would also be an LMI-we might as well dispense with any special structure, and resort to algebraic Riccati, bounded-real, or general LMI-based analysis, e.g., [16].

However, if $A$ has additional structure, for example, it has an embedded internally positive block transverse to the Lorentz cone axis, then the LMI (5) can be simplified. This simplification can be performed with diagonal dominance.

\section{B. Diagonal dominance.}

A square matrix $A \in \mathbf{R}^{n \times n}$ is (weakly) diagonally dominant if its entries satisfy

$$
\left|A_{i i}\right| \geq \sum_{j \neq i}\left|A_{i j}\right|, \quad \text { for all } i=1, \ldots, n .
$$

As a simple consequence of Gershgorin's circle theorem, diagonally dominant matrices with nonnegative diagonal entries are positive semidefinite.

If there exists a positive diagonal matrix $D \in \mathbf{R}^{n \times n}$ such that $A D$ is diagonally dominant, then the matrix $A$ is generalized or scaled diagonally dominant. Equivalently, there exists a positive vector $y \succ_{\mathbf{R}_{+}^{n}} 0$ such that

$$
\left|A_{i i}\right| y_{i} \geq \sum_{j \neq i}\left|A_{i j}\right| y_{j}, \quad \text { for all } i=1, \ldots, n \text {. }
$$

Note that generalized diagonally dominant matrices are also positive semidefinite, and include diagonally dominant matrices as a special case. Symmetric generalized diagonally dominant matrices with nonnegative diagonal entries are also known as $\mathrm{H}^{+}$-matrices, and have a very nice characterization as matrices with factor width of at most two, see [17, Theorem 9].

One consequence of this characterization is that $\mathrm{H}^{+}$ matrices can be written as a sum of positive semidefinite matrices that are nonzero only on a $2 \times 2$ principal submatrix, see [18]. For example, a $3 \times 3 H^{+}$-matrix $A=A^{T}$ is the sum of terms of the form

$$
A=\left[\begin{array}{ccc}
x_{1} & x_{2} & 0 \\
x_{2} & x_{3} & 0 \\
0 & 0 & 0
\end{array}\right]+\left[\begin{array}{ccc}
y_{1} & 0 & y_{2} \\
0 & 0 & 0 \\
y_{2} & 0 & y_{3}
\end{array}\right]+\left[\begin{array}{ccc}
0 & 0 & 0 \\
0 & z_{1} & z_{2} \\
0 & z_{2} & z_{3}
\end{array}\right],
$$

where the sub-matrices are all positive semidefinite,

$$
\left[\begin{array}{ll}
x_{1} & x_{2} \\
x_{2} & x_{3}
\end{array}\right] \succeq 0, \quad\left[\begin{array}{ll}
y_{1} & y_{2} \\
y_{2} & y_{3}
\end{array}\right] \succeq 0, \quad\left[\begin{array}{ll}
z_{1} & z_{2} \\
z_{2} & z_{3}
\end{array}\right] \succeq 0 .
$$

Recently, this fact has been exploited in [19], [20] to extend the reach of sum-of-squares techniques to high dimensional dynamical systems without imposing full LMI constraints on the Gram matrix, and in [18] to preprocess SDPs for numerical stability.

\section{Rotated quadratic constraints.}

Real, symmetric, positive semidefinite matrices of size $2 \times 2$, as they occur in the characterization of $\mathrm{H}^{+}$-matrices above, are special, because they satisfy a restricted hyperbolic constraint; hence their definiteness can be enforced with an SOCP rather than SDP [7]. Specifically, we have,

$$
\begin{aligned}
{\left[\begin{array}{ll}
x_{1} & x_{2} \\
x_{2} & x_{3}
\end{array}\right] \succeq 0 } & \Longleftrightarrow x_{1} \geq 0, \quad x_{3} \geq 0, \quad x_{1} x_{3}-x_{2}^{2} \geq 0 \\
& \Longleftrightarrow\left\|\left[\begin{array}{c}
2 x_{2} \\
x_{1}-x_{3}
\end{array}\right]\right\|_{2} \leq x_{1}+x_{3} \\
& \Longleftrightarrow\left(x_{1}+x_{3}, 2 x_{2}, x_{1}-x_{3}\right) \in \mathcal{L}_{+}^{3},
\end{aligned}
$$

for scalars $x_{1}, x_{2}$, and $x_{3}$. 


\section{EXAMPLES}

\section{A. Embedded shearless positive block.}

We show how these techniques can be used to consider the simple, augmented $(n+1)$-dimensional dynamics

$$
\left[\begin{array}{c}
\dot{x}_{0} \\
\dot{x}_{1}
\end{array}\right]=\left[\begin{array}{cc}
a_{0} & 0 \\
0 & A
\end{array}\right]\left[\begin{array}{c}
x_{0} \\
x_{1}
\end{array}\right], \quad a_{0} \in \mathbf{R}, \quad A \in \mathbf{R}^{n \times n},
$$

with trajectory $x(t)=\left(x_{0}(t), x_{1}(t)\right) \in \mathbf{R} \times \mathbf{R}^{n}$, on the cone $\mathcal{L}_{+}^{n+1}$. After writing out the LMI (5) in block form, we determine that the augmented system is $\mathcal{L}_{+}^{n+1}$-invariant if and only if

$$
2 a_{0} I-\left(A+A^{T}\right) \succeq 0 .
$$

It is stable if and only if $A$ is Hurwitz and $a_{0}<0$. Roughly speaking it is $\mathcal{L}_{+}^{n+1}$-invariant and stable if the stability degree of $A$ (i.e., minus the maximum real part of the eigenvalues of $A$ ) is at least $\left|a_{0}\right|$. See Figure 3 .

In general, the stability degree constraint (6) is an LMI in the variables $\left(A, a_{0}\right)$, however, if $A$ is Metzler then the LMI can be replaced with the constraint

$$
2 a_{0} I-\left(A+A^{T}\right) \text { is an } H^{+} \text {-matrix, }
$$

without any loss. From previous sections, the $\mathrm{H}^{+}$-matrix constraint (7) is an SOCP.

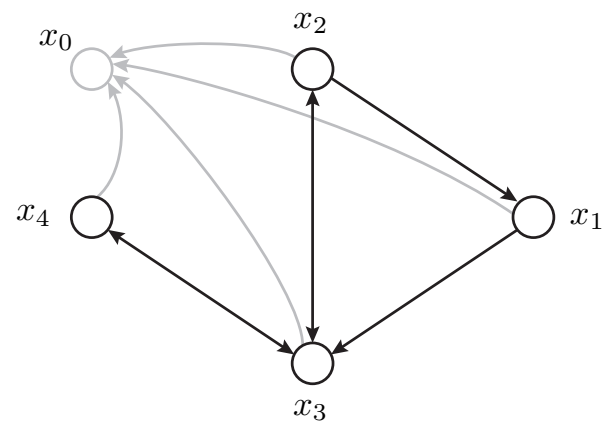

Fig. 2. Transportation network $x_{1}, \ldots, x_{4}$ from [3, Figure 2], augmented with a catch-all buffer $x_{0}$.

\section{B. Augmented transportation network.}

We consider the linear transportation network shown in Figure 2, which is inspired by the one studied in [3]. The network might represent a base system of four buffers (solid nodes $x_{1}, \ldots, x_{4}$ ) exchanging and consuming material in a way that preserves the network structure. The base system is augmented with an extra catch-all buffer (grayed out node $x_{0}$ ), leading to the augmented dynamics

$$
\left[\begin{array}{c}
\dot{x}_{0} \\
\dot{\bar{x}}
\end{array}\right]=\left[\begin{array}{cc}
a_{0} & h^{T} \\
0 & A
\end{array}\right]\left[\begin{array}{c}
x_{0} \\
\bar{x}
\end{array}\right], \quad x_{0}(t) \in \mathbf{R}, \quad \bar{x}(t) \in \mathbf{R}^{4},
$$

where the base dynamics $\dot{\bar{x}}=A \bar{x}$ are given by the internally positive system

$$
\underbrace{\left[\begin{array}{c}
\dot{x}_{1} \\
\dot{x}_{2} \\
\dot{x}_{3} \\
\dot{x}_{4}
\end{array}\right]}_{\dot{\bar{x}}(t)}=\underbrace{\left[\begin{array}{cccc}
-1-\ell_{31} & \ell_{12} & 0 & 0 \\
0 & -\ell_{12}-\ell_{32} & \ell_{23} & 0 \\
\ell_{31} & \ell_{32} & -\ell_{23}-\ell_{43} & \ell_{34} \\
0 & 0 & \ell_{43} & -4-\ell_{34}
\end{array}\right]}_{A} \underbrace{\left[\begin{array}{l}
x_{1} \\
x_{2} \\
x_{3} \\
x_{4}
\end{array}\right]}_{\bar{x}(t)} .
$$

Here, the state $x_{i}$ represents the amount of material at node $i, \ell_{i j} \geq 0$ the transfer rate between nodes $i$ and $j$ in the base system, $a_{0} \in \mathbf{R}$ the self-degradation rate of the catchall buffer, and $h_{i}$ the rate of transfer between node $i$ and the catch-all node. Note that $A$ is Metzler by construction, but the augmented system (8) need not be.

Several problems are now readily solved: by checking (6) (or (7)), with the $\ell_{i j}$ treated as variables and $h=0$ fixed, the augmented system is $\mathcal{L}_{+}^{5}$-invariant only if $a_{0} \geq-1.25$, which is an upper bound on the Metzler eigenvalue of $A$. Thus the catch-all node must consume material no faster than with rate constant 1.25. In addition, if $\ell_{i j}$ and $h_{i}$ are known, and the augmented system (8) is $\mathcal{L}_{+}^{5}$-invariant, then it is stable provided $a_{0}<0$. Of course, these types of problems can be cast as an LP or SOCP.

\section{Other examples on $\mathcal{L}_{+}^{n}$.}
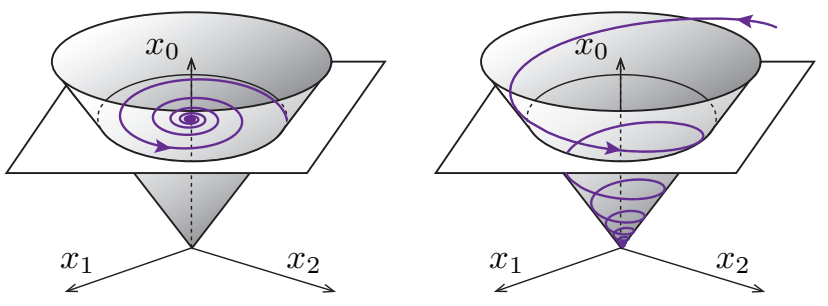

Fig. 3. Embedded focus along $x_{0}$-axis

1) Twist system: Suppose $A+A^{T}=0$ (skew-symmetric). The matrix system

$$
\left[\begin{array}{c}
\dot{x}_{0} \\
\dot{x}_{1}
\end{array}\right]=\left[\begin{array}{cc}
a_{0} & h^{T} \\
0 & A
\end{array}\right]\left[\begin{array}{l}
x_{0} \\
x_{1}
\end{array}\right],
$$

is $\mathcal{L}_{+}^{n}$-invariant if and only if $\|h\|_{2} \leq a_{0}$, i.e., the point $\left(a_{0}, h\right)$ is in the Lorentz cone $\mathcal{L}_{+}^{n}$. Note if $a_{0}=0$, the dynamics correspond to a twist in homogeneous coordinates, see, e.g., [21].

2) Proper orthochronous Lorentz transformations: The restricted Lorentz group $S^{+}(1, n-1)$ is generated by spatial rotations and Lorentz boosts, and consists of linear transformations that keep the quadratic form $x^{T} J_{n} x$ invariant. In particular, elements of $S O^{+}(1, n-1)$ correspond to $\mathcal{L}_{+}^{n}$-invariant dynamics matrices, and thus fall within our framework.

\section{CONCLUSION}

In this work we analyzed linear systems with a special structure by Jordan algebraic techniques. In particular, we put analysis of internally positive systems, which have recently been in vogue, in the same theoretical framework as systems that are exponentially invariant with respect to the Lorentz cone, as well as general linear systems. It is evident that Lyapunov functions for such systems can be significantly simpler, computationally and representationally, than if the special cone-invariant dynamic structure were not exploited. In this framework, summarized in Table I, the relevant structure is cross-positivity of the dynamics matrix on a symmetric cone. 


\begin{tabular}{r|c|c|c}
\hline Algebra: & Real & Lorentz & Symmetric \\
\hline$V$ & $\mathbf{R}^{n}$ & $\mathbf{R}^{n}$ & $\mathbf{S}^{n}$ \\
$K$ & $\mathbf{R}_{+}^{n}$ & $\mathcal{L}_{+}^{n}$ & $\mathbf{S}_{+}^{n}$ \\
$\langle x, y\rangle$ & $x^{T} y$ & $x^{T} y$ & $\operatorname{Tr}\left(X Y^{T}\right)$ \\
$x \circ y$ & $x_{i} y_{i}$ & $\left(x^{T} y, x_{0} y_{1}+y_{0} x_{1}\right)$ & $\frac{1}{2}(X Y+Y X)$ \\
$P_{z}, z \in \operatorname{int} K$ & $\operatorname{diag}(z)^{2}$ & $z z^{T}-\frac{z^{T} J_{n} z}{2^{2}} J_{n}$ & $X \mapsto Z X Z$ \\
$V(x)=\left\langle x, P_{z}(x)\right\rangle$ & $x^{T} \operatorname{diag}(z)^{2} x$ & $x^{T}\left(z z^{T}-\frac{z^{T} J_{n} z}{2} J_{n}\right) x$ & $\|X Z\|_{F}^{2}$ \\
Free variables in $V(x)$ & $n$ & $n$ & $n(n+1) / 2$ \\
dynamics $L$ & $x \mapsto A x$ & $x \mapsto A x$ & $X \mapsto A X+X A^{T}$ \\
$L$ is cross-positive & $A$ is Metzler & $A$ satisfies $(5)$ & by construction \\
$-L(p) \succ_{K} 0$ & $(A p)_{i}<0$ & $\left\|(A p)_{1}\right\|_{2}<(-A p)_{0}$ & $A P+P A^{T} \prec 0$ \\
Stability verification & LP & SOCP & SDP \\
\hline
\end{tabular}

TABLE I

SUMMARY OF DYNAMICS PRESERVING A CONE

Unfortunately, while cross-positivity has a fairly simple LP characterization in terms of the corresponding dynamics matrix (via the Metzler structure) for internally positive systems, the same condition is generically much more complicated for $\mathcal{L}_{+}^{n}$-invariant dynamics: the condition is an LMI in the dynamics matrix. In fact, the condition corresponding to entrywise positivity of the dynamics matrix is also an LMI for discrete time systems [22], [23]. As a result, $\mathcal{L}_{+}^{n}$ invariance, by itself, is not computationally attractive for high dimensional systems without taking advantage of even more special structure. We gave a simple (almost trivial) example of how this might be done by augmenting an internally positive system with a catch-all block.

Future work will aim to apply these techniques to control synthesis and as well as to further study special structure. An interesting question is whether it is possible to verify stability of certain subclasses of, e.g., difference of positive or ellipsoidal cone invariant systems via LP or SOCP, rather than via SDP.

\section{ACKNOWLEDGMENTS}

We thank S. You, N. Matni, and A. Swaminathan for helpful discussions. This work was supported in part by a Department of Defense NDSEG Fellowship, Terraswarm, one of six centers of STARnet, a Semiconductor Research Corporation program sponsored by MARCO and DARPA, and by the Boeing company.

\section{REFERENCES}

[1] A. Berman and R. J. Plemmons, Nonnegative Matrices in the Mathematical Sciences, ser. Classics in Applied Mathematics. SIAM, 1994.

[2] P. D. Leenheer and D. Aeyels, "Stabilization of positive linear systems," Systems and Control Letters, vol. 44, no. 4, pp. 259-271, 2001.

[3] A. Rantzer, "Distributed control of positive systems," arXiv:1203.0047 [math.OC], Feb. 2012.

[4] K. J. Åström and R. M. Murray, Feedback Systems: An Introduction for Scientists and Engineers. Princeton University Press, 2008.

[5] S. P. Boyd and L. Vandenberghe, Convex Optimization. Cambridge University Press, 2004.
[6] R. T. Rockafellar, Convex Analysis. Princeton University Press, 1970

[7] F. Alizadeh and D. Goldfarb, "Second-order cone programming," Mathematical Programming, vol. 95, no. 1, pp. 3-51, 2003.

[8] J. Faraut and A. Korányi, Analysis on Symmetric Cones, ser. Oxford Mathematical Monographs. Oxford University Press, 1994.

[9] M. S. Gowda, R. Sznajder, and J. Tao, "Some $P$-properties for linear transformations on Euclidean Jordan algebras," Linear Algebra and its Applications, vol. 393, no. 0, pp. 203-232, 2004.

[10] M. S. Gowda and R. Sznajder, "Some global uniqueness and solvability results for linear complementarity problems over symmetric cones," SIAM Journal on Optimization, vol. 18, no. 2, pp. 461-481, 2007.

[11] L. Vandenberghe, "Symmetric cones," EE236C Lecture Notes, 2014.

[12] H. Schneider and M. Vidyasagar, "Cross-positive matrices," SIAM Journal on Numerical Analysis, vol. 7, no. 4, pp. 508-519, 1970.

[13] R. J. Stern and H. Wolkowicz, "Exponential nonnegativity on the ice cream cone," SIAM Journal on Matrix Analysis and Applications, vol. 12, no. 1, pp. 160-165, 1991.

[14] M. S. Gowda and J. Tao, " $Z$-transformations on proper and symmetric cones," Mathematical Programming, vol. 117, no. 1-2, pp. 195-221, 2009.

[15] C. Grussler and A. Rantzer, "Modified balanced truncation preserving ellipsoidal cone-invariance," in IEEE Conference on Decision and Control (CDC), Dec. 2014, pp. 2365-2370.

[16] S. P. Boyd, L. El Ghaoui, E. Feron, and V. Balakrishnan, Linear Matrix Inequalities in System and Control Theory, ser. Studies in Applied and Numerical Mathematics. SIAM, 1994, vol. 15.

[17] E. G. Boman, D. Chen, O. Parekh, and S. Toledo, "On factor width and symmetric $H$-matrices," Linear Algebra and its Applications, vol. 405, no. 0, pp. 239-248, 2005.

[18] F. Permenter and P. Parrilo, "Partial facial reduction: simplified, equivalent SDPs via approximations of the PSD cone," arXiv:1408.4685 [math.OC], Nov. 2014.

[19] A. A. Ahmadi and A. Majumdar, "DSOS and SDSOS optimization: LP and SOCP-based alternatives to sum of squares optimization," in Conference on Information Sciences and Systems (CISS), Mar. 2014, pp. $1-5$.

[20] A. Majumdar, A. A. Ahmadi, and R. Tedrake, "Control and verification of high-dimensional systems with DSOS and SDSOS programming," in IEEE Conference on Decision and Control (CDC), Dec. 2014, pp. 394-401.

[21] R. M. Murray, Z. Li, and S. S. Sastry, A Mathematical Introduction to Robotic Manipulation. CRC Press, 1994.

[22] R. Hildebrand, "An LMI description for the cone of Lorentz-positive maps," Linear and Multilinear Algebra, vol. 55, no. 6, pp. 551-573, 2007.

[23] —_ "An LMI description for the cone of Lorentz-positive maps II," Linear and Multilinear Algebra, vol. 59, no. 7, pp. 719-731, 2011. 